TRANSACTIONS OF THE

AMERICAN MATHEMATICAL SOCIETY

Volume 361, Number 3, March 2009, Pages 1333-1349

S 0002-9947(08)04710-7

Article electronically published on October 14, 2008

\title{
CONSTRUCTION OF GLOBAL FUNCTION FIELDS FROM LINEAR CODES AND VICE VERSA
}

\author{
CHAOPING XING AND SZE LING YEO
}

\begin{abstract}
We introduce a new connection between linear codes and global function fields, which in turn allows us to construct new global function fields with improved lower bounds on the number of rational places. The genus and number of rational places of subfields of certain families of cyclotomic function fields are given as well.
\end{abstract}

\section{INTRODUCTION}

The inter-relationship between error-correcting codes and global function fields with many rational places was discovered as early as the 1980s with the invention of Goppa geometric codes [7, 8, 9. In fact, the introduction of Goppa geometric codes was primarily responsible for an increased interest among researchers to seek for global function fields with large numbers of rational places. From the perspective of algebraic geometry, this is equivalent to the construction of smooth, projective, absolutely irreducible curves over finite fields with many rational points, which is itself an interesting research problem motivated by the initial work of Weil in the 1940s.

Apart from Goppa geometric codes, other code constructions based on global function fields with many rational places, including algebraic geometric codes and their variations, were subsequently proposed by various researchers [5, 6, 17, 21, 26, 24, 25. Just like their predecessor, a number of these code constructions are interesting as they provide good asymptotic results.

In this paper, we present yet another method to show how linear codes and global function fields are intertwined. In a way, this construction differs from the previous constructions since it partly arises out of the search for global function fields with a large number of rational places. More specifically, given a global function field $F / \mathbb{F}_{q}$ for a prime power $q$ and a narrow ray class field extension $F^{\prime} / F$, we fix a set of places $S$ of $F$. We can then construct a linear code whose length is $|S|$ and whose dimension is the $p$-rank of the Galois group $\operatorname{Gal}\left(F^{\prime} / F\right)$, where $p$ is the characteristic of $\mathbb{F}_{q}$. Information about the minimum distance $d$ of the code in turn allows us to construct a subfield $L$ of $F^{\prime}$ in which the number of rational places it contains is closely related to $d$. Further, by applying the theory of global function

Received by the editors September 1, 2005 and, in revised form, January 4, 2007.

2000 Mathematics Subject Classification. Primary 11G20, 14H05, 11R60.

The first author is the corresponding author.

The first author was partially supported by the National Scientific Research Project 973 of China 2004CB318000. 
fields again, we can investigate the parameters of the dual codes with respect to the codes constructed above.

In the next section, we briefly review some of the main concepts of global function fields including properties of ray class fields and narrow ray class fields. We then present our code construction and the associated global function field in Section 3. In Section 4, we discuss some explicit examples of codes constructed in this way. Next, we illustrate in Section 5 how we can use this method to construct global function fields having many rational places. Finally in Section 6, we apply this construction to certain families of cyclotomic function fields. In particular, we are able to determine the genera and splitting behaviour of places of $F$ in some subfields of these cyclotomic function fields. Together with the aid of mathematical software packages, a couple of new global function fields with improved lower bounds on the number of rational places are therefore obtained.

Throughout this paper, we frequently refer to the interactive website maintained by Brouwer [4] for bounds on the parameters of various linear codes. As far as we are aware, this website contains the most up-to-date repository of results for the bounds on the minimum distances of linear codes, especially for binary and ternary linear codes. On the other hand, a table for bounds on the number of rational points for function fields is regularly updated by van der Geer and van der Vlugt 22. At the time of this writing, Table 17 is the latest version and serves as the basis for all our comparisons.

\section{RAY CLASS FIELDS AND NARROW RAY CLASS FIELDS}

For most of the results in this section, the reader may refer to $19,15,1,3$.

We begin with some notation. For a prime number $p$, let $q=p^{r}$ and consider the global function field $F / \mathbb{F}_{q}$. A place $P$ of $F$ is said to be rational if its residue class field $\tilde{F}_{P}=\mathcal{O}_{P} / P$ is isomorphic to $\mathbb{F}_{q}$, where $\mathcal{O}_{P}$ is the valuation ring of $P$. Let $N(F)$ be the number of rational places of $F$. According to the Hasse-Weil bound, if $g(F)$ is the genus of $F$, then $N(F)$ must satisfy

$$
N(F) \leq q+1+2 g(F) q^{1 / 2} .
$$

Several improvements to the Hasse-Weil bound have been achieved. An up-todate survey for the upper and lower bounds on $N_{q}(g)$, or the maximum number of rational places that a global function field $F / \mathbb{F}_{q}$ of genus $g$ can attain, is maintained by van der Geer and van der Vlugt 22.

Throughout this paper, we shall assume that $N(F) \geq 1$ and distinguish one such rational place $\infty$ of $F$. Further, we fix a place $P$ of $F$ of degree $d$ and let $D=n P$ be a divisor of $F$ for some nonnegative integer $n$. Let $\mathcal{S}_{\infty}$ denote the set of all the places of $F$ with $\infty$ omitted and let $\mathcal{S}$ be a subset of $\mathcal{S}_{\infty}$ containing $P$ such that $\mathcal{S}_{\infty}-\mathcal{S}$ is finite.

Recall that $\mathcal{O}_{\mathcal{S}}$ is the integral ring consisting of the functions of $F$ which are regular at all the places in $\mathcal{S}$. For any place $Q \in \mathcal{S}$, we will also denote by $Q$ the prime ideal $Q \cap \mathcal{O}_{\mathcal{S}}$ and thus, the divisor $n P$ can be identified with the ideal $P^{n}$. Let $I_{D}(\mathcal{S})$ be the group of the fractional ideals of $\mathcal{O}_{\mathcal{S}}$ that are prime to $P$ and $\operatorname{Princ}_{D}(\mathcal{S})$ be a subgroup of $I_{D}(\mathcal{S})$ comprising principal ideals of $\mathcal{O}_{\mathcal{S}}$. In particular, if $z \mathcal{O}_{\mathcal{S}}$ is in $\operatorname{Princ}_{D}(\mathcal{S})$, then $\nu_{P}(z-1) \geq n$. The factor group $\mathrm{Cl}_{D}\left(\mathcal{O}_{\mathcal{S}}\right)$ is called the $\mathcal{S}$-ray class group with modulus $D$. The $\mathcal{S}$-ray class group with modulus 0 is more commonly known as the $\mathcal{S}$-ideal class group and is simply denoted by $\mathrm{Cl}\left(\mathcal{O}_{\mathcal{S}}\right)$. 
Similarly, let $\operatorname{Div}_{D}^{0}(F)$ be the group of divisor classes of $F$ which are of the form $X-(\operatorname{deg} X) \infty$ such that $P$ is not in the support of $X$ and let $\operatorname{Princ}_{D}(F)$ be a subgroup of $\operatorname{Div}_{D}^{0}(F)$ consisting of principal divisors $\operatorname{div}(z)$ in which $\nu_{P}(z-1) \geq n$. We can then define the factor group $\mathrm{Cl}_{D}(F)$ as the quotient $\operatorname{Div}_{D}^{0}(F) / \operatorname{Princ}_{D}(F)$. Once again, if $n=0$, we obtain our usual divisor class group $\mathrm{Cl}(F)=\operatorname{Div}^{0}(F) / \operatorname{Princ}(F)$ whose order, denoted by $h(F)$, is finite and is called the class number of $F$.

The above groups are related according to the following proposition.

Proposition 2.1. (i) $\mathrm{Cl}_{D}\left(\mathcal{O}_{\mathcal{S}}\right)$ is isomorphic to $\operatorname{Div}_{D}^{0}(F) /\left(\operatorname{Div}_{\mathcal{S}}^{0}(F)+\operatorname{Princ}_{D}(F)\right)$, where $\operatorname{Div}_{\mathcal{S}}^{0}(F)$ is the group of divisor classes of degree 0 whose support is disjoint from $\mathcal{S}$.

(ii) We have the following exact sequence:

$$
\begin{aligned}
0 \rightarrow \operatorname{Div}_{\mathcal{S}}^{0}(F) /\left(\operatorname{Div}_{\mathcal{S}}^{0}(F) \cap \operatorname{Princ}_{D}(F)\right) & \rightarrow \operatorname{Div}_{D}^{0}(F) / \operatorname{Princ}_{D}(F) \\
& \rightarrow \operatorname{Div}_{D}^{0}(F) /\left(\operatorname{Div}_{\mathcal{S}}^{0}(F)+\operatorname{Princ}_{D}(F)\right) .
\end{aligned}
$$

In particular, $\mathrm{Cl}_{D}\left(\mathcal{O}_{\mathcal{S}_{\infty}}\right) \cong \mathrm{Cl}_{D}(F)$.

By (ii) of Proposition 2.1, we see that every fractional ideal of $\mathrm{Cl}_{D}\left(\mathcal{O}_{\mathcal{S}}\right)$ can be identified with a divisor class of $\mathrm{Cl}_{D}(F)$. More precisely, the ideal $\prod_{Q \neq P, \infty} Q^{m_{Q}}$ is identified with $\sum_{Q \neq P, \infty} m_{Q}(Q-\operatorname{deg} Q \infty)$. Using this identification, it follows immediately that

$$
\mathrm{Cl}_{D}\left(\mathcal{O}_{\mathcal{S}}\right) \cong \mathrm{Cl}_{D}\left(\mathcal{O}_{\mathcal{S}_{\infty}}\right) /\left\langle\mathcal{S}^{*}\right\rangle_{n}
$$

where $\mathcal{S}^{*}=\mathcal{S}_{\infty}-\mathcal{S}$ and $\left\langle\mathcal{S}^{*}\right\rangle_{n}$ is the subgroup of $\mathrm{Cl}_{D}\left(\mathcal{O}_{\infty}\right)=\mathrm{Cl}_{n P}\left(\mathcal{O}_{\infty}\right)$ generated by all the places of $\mathcal{S}^{*}$.

For the set $\mathcal{S}$, we recall that a ray class field with modulus $D$ is constructed as the finite abelian field extension of $F$ corresponding to a certain open subgroup of the idèle class group of $F$ of finite index in which the Galois group is isomorphic to $\mathrm{Cl}_{D}\left(\mathcal{O}_{\mathcal{S}}\right)$ (refer to [15]). Such a ray class field with modulus $D$ is denoted by $F_{\mathcal{S}}^{D}$. We summarize the main properties of $F_{\mathcal{S}}^{D}$ in the theorem below.

Theorem 2.2 (Properties of ray class fields). (i) $F_{\mathcal{S}}^{D}$ is the largest finite abelian extension $F^{\prime}$ of $F$ in which all places that are not in $\mathcal{S}$ split completely in $F^{\prime} / F$ and the conductor of $F^{\prime} / F \leq D$.

(ii) The Galois group $\operatorname{Gal}\left(F_{\mathcal{S}}^{D} / F\right) \cong \mathrm{Cl}_{D}\left(\mathcal{O}_{\mathcal{S}}\right)$.

(iii) All places of $F$ different from $P$ are unramified in $F_{\mathcal{S}}^{D} / F$ and for each such place $Q$, its Artin symbol corresponds to the residue class of $Q$ in $\mathrm{Cl}_{D}\left(\mathcal{O}_{\mathcal{S}}\right)$ under the correspondence in (ii).

(iv) The full constant field of $F_{\mathcal{S}}^{D}$ is $\mathbb{F}_{q}$.

(v) For an integer $-1 \leq i \leq n$, the subfield $F^{i}$ of $F_{\mathcal{S}}^{D}$ fixed by the $i$-th upper ramification group of $P$ (see [18, for the definition) is $F_{\mathcal{S}}^{i P}$.

Remark 2.3. (i) Parts 1, 2 and 4 are true for any effective divisor $D$; i.e., we do not have to assume that $D=n P$.

(ii) In the case when $D=0$, the ray class field $H_{\mathcal{O}_{\mathcal{S}}}=F_{\mathcal{S}}^{0}$ is called the $\mathcal{S}$-Hilbert class field. It is characterized by the property that it is the maximum unramified abelian extension $F^{\prime}$ of $F$ in which every place of $F$ that is not in $\mathcal{S}$ splits completely in $F^{\prime} / F$.

From (v) of Theorem 2.2 and the upper index form of the Hilbert different formula [1, 3], it follows that the different exponent of $P$ with respect to the extension 
$F_{\mathcal{S}}^{D}$ is given by

$$
d_{P}=d\left(\left[F_{\mathcal{S}}^{n P}: F_{\mathcal{S}}^{0}\right] n-\sum_{i=0}^{n-1}\left[F_{\mathcal{S}}^{i P}: F_{\mathcal{S}}^{0}\right]\right) .
$$

Thus, the Hurwitz genus formula applied to $F_{\mathcal{S}}^{D} / F$ yields

$$
2 g\left(F_{\mathcal{S}}^{D}\right)-2=\left[F_{\mathcal{S}}^{D}: F\right](2 g(F)-2+n d)-d \sum_{i=0}^{n-1}\left[F_{\mathcal{S}}^{i P}: F\right] .
$$

From $\left[F_{\mathcal{S}}^{i P}: F\right]=\left|\mathrm{Cl}_{i P}\left(\mathcal{O}_{\mathcal{S}}\right)\right|$ for $i=0, \ldots, n-1$, and (1), the genus of $F_{\mathcal{S}}^{D}$ can be reformulated as:

$$
2 g\left(F_{\mathcal{S}}^{D}\right)-2=\frac{\left|\mathrm{Cl}_{D}\left(\mathcal{O}_{\mathcal{S}_{\infty}}\right)\right|}{\left|\left\langle\mathcal{S}^{*}\right\rangle_{i}\right|}(2 g(F)-2+n d)-d \sum_{i=0}^{n-1} \frac{\left|\mathrm{Cl}_{i P}\left(\mathcal{O}_{\mathcal{S}_{\infty}}\right)\right|}{\left|\left\langle\mathcal{S}^{*}\right\rangle_{i}\right|} .
$$

In what follows, let $A=\mathcal{O}_{\mathcal{S}_{\infty}}$. We next wish to know more about the structure of $\mathrm{Cl}_{D}(A)$. To do that, we will consider the narrow ray class field with modulus $D$. First, we need the following lemma:

Lemma 2.4. Let $F_{\infty}$ be the $\infty$-adic completion of $F$ at $\infty$. There is a sign function sgn: $F_{\infty}^{*} \longrightarrow \mathbb{F}_{q}^{*}$ which is a multiplicative group homomorphism on $F_{\infty}^{*}$ such that:

(i) $\operatorname{sgn}(\alpha)=\alpha$ for any $\alpha \in \mathbb{F}_{q}^{*}$;

(ii) $\operatorname{sgn}\left(U_{\infty}^{(1)}\right)=\{1\}$, where $U_{\infty}^{(1)}$ consists of the functions $z$ in $F$ such that $\nu_{\infty}(z-1) \geq 1$.

Fix a sign function sgn. Let $\operatorname{Princ}_{D}^{+}\left(\mathcal{S}_{\infty}\right)$ be the group of principal ideals $z A$, where $\nu_{P}(z-1) \geq n$ and $\operatorname{sgn}(z)=1$. The factor group $I_{D}\left(\mathcal{S}_{\infty}\right) / \operatorname{Princ}_{D}^{+}\left(\mathcal{S}_{\infty}\right)$, which we denote by $C \ell_{D}^{+}(A)$, is called the narrow ray class group with modulus $D$ with respect to the sign function sgn. The narrow ray class field with modulus $D$, denoted by $F^{D}(\infty)$, is constructed as the finite abelian field extension of $F$ corresponding to an open subgroup of the idèle class group of $F$ such that its Galois group $\operatorname{Gal}\left(F^{D}(\infty) / F\right) \cong C \ell_{D}^{+}(A)$ [15]. In fact, $F^{D}(\infty)$ is a finite extension of $F_{\mathcal{S}_{\infty}}^{D}$ satisfying the following properties:

Theorem 2.5 (Main properties of narrow ray class fields).

(i)

(ii)

$$
\operatorname{Gal}\left(F^{D}(\infty) / F\right) \cong C \ell_{D}^{+}(A)
$$

$$
\left[F^{D}(\infty): F_{\mathcal{S}_{\infty}}^{D}\right]=q-1 .
$$

(iii) $F_{\mathcal{S}_{\infty}}^{D}$ is both the decomposition field and the fixed field of $\infty$ in $F^{D}(\infty)$. In particular,

$$
\operatorname{Gal}\left(F^{D}(\infty) / F_{\mathcal{S}_{\infty}}^{D}\right) \cong \mathrm{Cl}_{D}(A) / C \ell_{D}^{+}(A) \cong \mathbb{F}_{q}^{*} .
$$

(iv) All places of $F$ apart from $P$ and $\infty$ are unramified in $F^{D}(\infty) / F$. For such a place $Q$, its Artin symbol is given by the residue class in $C \ell_{D}^{+}(A)$ under the correspondence in (i).

(v) The conductor of $F^{D}(\infty) / F$ is $n P+\min (q-2,1) \infty$.

(vi) $\mathbb{F}_{q}$ is the full constant field of $F^{D}(\infty) / F$. 
Alternatively, $F^{D}(\infty)$ can be constructed via an sgn-normalized Drinfeld module of rank 1 [1], 12. Suppose that $H_{A}$ is the $\mathcal{S}_{\infty}$-Hilbert class field of $F$. Then, according to [1], we have $\operatorname{Gal}\left(F^{D}(\infty) / H_{A}\right) \cong\left(A / P^{n}\right)^{*}$. Hence, $\left(A / P^{n}\right)^{*}$ can be identified with a subgroup of $C \ell_{D}^{+}(A)$. Moreover, for every place $P^{\prime}$ of $H_{A}$ lying over $P, P^{\prime}$ is totally ramified in $F^{D}(\infty) / H_{A}$. Since it follows from (11) that $|\mathrm{Cl}(A)|=$ $h(F)$, we have

$$
\left[F^{D}(\infty): F\right]=\left[F^{D}(\infty): H_{A}\right]\left[H_{A}: F\right]=h(F) \Phi\left(P^{n}\right),
$$

where $\Phi\left(P^{n}\right)=\left|\left(A / P^{n}\right)^{*}\right|=\left(q^{d}-1\right) q^{d(n-1)}$.

Theorem 2.6. Let $G_{D}$ be the subgroup of $C \ell_{D}^{+}(A)$ generated by $\mathbb{F}_{q}^{*}$ and the places in $\mathcal{S}^{*}$. Let $L$ be the subfield of $F^{D}(\infty)$ fixed by $G_{D}$. Then $L$ has at least $\frac{h(F) \Phi\left(P^{n}\right)\left(\left|\mathcal{S}^{*}\right|+1\right)}{\left|G_{D}\right|}$ rational places. The genus of $L$ is given by

$$
2 g(L)-2=h(F)\left[\frac{\Phi\left(P^{n}\right)}{\left|G_{D}\right|}(2 g(F)-2+n d) d \sum_{i=0}^{n-1} \frac{\Phi\left(P^{i}\right)}{\left|G_{P^{i}}\right|}\right] .
$$

Proof. By the fact that $\mathbb{F}_{q}{ }^{*}$ is the decomposition group of $\infty$ in $F^{\prime}$ and by consideration of the Artin symbols of the places in $\mathcal{S}^{*}$, it is clear that $\infty$ and the places in $\mathcal{S}^{*}$ split completely in $L / F$. Hence, we conclude from the characterization of $F_{\mathcal{S}}^{D}$ that $L$ is a subfield of the ray class field $F_{\mathcal{S}}^{D}$. Further since $C \ell_{D}^{+}(A) / G_{D} \cong \mathrm{Cl}_{D}(A) /\left\langle\mathcal{S}^{*}\right\rangle_{n}$, it follows from 1 that $L$ is indeed the ray class field $F_{\mathcal{S}}^{D}$. The genus is now obtained via (2).

\section{The MAin CONSTRUCtion}

In this section, we will introduce the main idea of our construction. First of all, let us establish some preliminary lemmas.

Lemma 3.1. The p-rank of the abelian group $\left(A / P^{n}\right)^{*}$ is $r d(n-1-\lfloor(n-1) / p\rfloor)$, where $\lfloor x\rfloor$ is the largest integer $\leq x$.

Proof. Clearly, $A / P^{n} \cong \mathcal{O}_{P} / P^{n}=\left\{\sum_{i=0}^{n-1} a_{i} \pi^{i} \bmod P^{n}: a_{i} \in \tilde{F}_{P}, 0 \leq i \leq n-1\right\}$, where $\pi$ is a local parameter at $P$. Hence,

$$
\left(A / P^{n}\right)^{*}=\left\{\sum_{i=0}^{n-1} a_{i} \pi^{i} \bmod P^{n}: a_{i} \in \tilde{F}_{P}, 0 \leq i \leq n-1, a_{0} \neq 0\right\}
$$

and has cardinality $\left(q^{d}-1\right) q^{d(n-1)}$. Further,

$$
\begin{aligned}
\left(\left(A / P^{n}\right)^{*}\right)^{p} & =\left\{\sum_{i=0}^{n-1} a_{i} \pi^{i p} \bmod P^{n}: a_{i} \in \tilde{F}_{P}, 0 \leq i \leq n-1, a_{0} \neq 0\right\} \\
& =\left\{\sum_{i=0}^{n^{\prime}} a_{i} \pi^{i p} \bmod P^{n}: a_{i} \in \tilde{F}_{P}, 0 \leq i \leq n^{\prime}, a_{0} \neq 0\right\},
\end{aligned}
$$

where $n^{\prime}=\lfloor(n-1) / p\rfloor$. This gives $\left|\left(\left(A / P^{n}\right)^{*}\right)^{p}\right|=\left(q^{d}-1\right) q^{d n^{\prime}}$, which implies that

$$
\left|\left(A / P^{n}\right)^{*} /\left(\left(A / P^{n}\right)^{*}\right)^{p}\right|=q^{d\left(n-1-n^{\prime}\right)} .
$$

Consequently, the dimension of the vector space $\left(A / P^{n}\right)^{*} /\left(\left(A / P^{n}\right)^{*}\right)^{p}$ is $r d\left(n-1-n^{\prime}\right)$ as desired. 
Our next lemma shows how the minimum distance of a linear code can be determined from the generator matrix and the parity-check matrix of the code.

Lemma 3.2. Let $C$ be an $[N, \kappa]$-linear code over $\mathbb{F}_{q}$ and let $\mathcal{G}$ and $\mathcal{H}$ be the generator matrix and parity-check matrix of $C$, respectively.

(i) $C$ has minimum distance $\delta$ if and only if every $\delta-1$ columns of $\mathcal{H}$ are linearly independent and there exist $\delta$ columns of $\mathcal{H}$ that are linearly dependent.

(ii) $C$ has minimum distance $\delta$ if and only if every $N-\delta+1$ columns of $\mathcal{G}$ have rank $\kappa$ and there exist $N-\delta$ columns of $\mathcal{G}$ of rank $\kappa-1$.

Proof. (i) This is a standard result in coding theory (see, for example, 14]).

(ii) Suppose that the minimum distance of $C$ is $\delta$. We first show that there exist $N-\delta$ columns of $\mathcal{G}$ of rank $\kappa-1$. Let $\mathbf{c}$ be a codeword of $C$ of weight $\delta$. Let $C_{\mathbf{c}}$ be the residual code with respect to $\mathbf{c}$ obtained from $C$ by deleting from all the codewords in $C$ the coordinates in the support of $\mathbf{c}$. Then, it follows from [14, Lemma 5.7.3] that $C_{\mathbf{c}}$ has dimension $\kappa-1$. Since the submatrix obtained from $\mathcal{G}$ by deleting the columns of $\mathcal{G}$ corresponding to the coordinates in the support of $\mathbf{c}$ is a matrix whose rows generate the code $C_{\mathbf{c}}$, the $N-\delta$ columns in this submatrix must have rank $\kappa-1$. Conversely, suppose that every $N-\delta+1$ columns of $\mathcal{G}$ have rank $\kappa$ and there exist $N-\delta$ columns of $\mathcal{G}$ of rank $\kappa-1$. Without loss of generality, we assume that these columns are from the first $N-\delta$ columns of $\mathcal{G}$. Write $\mathcal{G}=\left[\mathcal{G}^{\prime} \mathbf{u}_{N-\delta+1} \ldots \mathbf{u}_{N}\right]$, where $\gamma^{\prime}$ consists of the first $N-\delta$ columns of $\mathcal{G}$. Since $\mathcal{G}^{\prime}$ has rank $\kappa-1$ and $\mathcal{G}^{\prime}$ has $\kappa$ rows, there exists a nonzero $\mathbf{v}=\left(v_{1}, \ldots, v_{\kappa}\right)$ such that

$$
\mathbf{v} \mathcal{G}^{\prime}=0 \text {. }
$$

Thus, $\nu \mathcal{G}$ is a codeword of $C$ of weight at most $\delta$. If $d(C)<\delta$, the first part of our proof implies that there must be some $N-\delta+1$ columns of $\mathcal{G}$ of rank $\kappa-1$, contradicting our assumption. Consequently, $d(C)=\delta$. It remains to show that if $d(C)=\delta$, every $N-\delta+1$ columns of $\mathcal{G}$ have rank $\kappa$, but this is immediate from what we have just proven.

Now, let $F^{\prime}=F^{D}(\infty)$ be the narrow ray class field with modulus $D=n P$. Let $V_{D}$ be the $\mathbb{F}_{p}$-vector space obtained by taking the quotient of $C \ell_{D}^{+}(A)$ by its maximal abelian subgroup, i.e.

$$
V_{D}=C \ell_{D}^{+}(A) / C \ell_{D}^{+}(A)^{p}
$$

Since the $p$-rank of an abelian group is also equal to the number of summands in the direct product of its Sylow $p$-subgroup into cyclic components, the dimension $\kappa$ of $V_{D}$ is at least as large as that of $(A / D)^{*} /\left((A / D)^{*}\right)^{p}$ which, by Lemma 3.1 is $r d(n-1-\lfloor(n-1) / p\rfloor)$.

Let $S$ be a finite subset of $\mathcal{S}_{\infty}-\{P\}$, say $S=\left\{P_{1}, P_{2}, \ldots, P_{N}\right\}$. Clearly, each element of $S$ can be viewed as a $\kappa$-vector in $V_{D}$. Write each element of $S$ as a $\kappa$ tuple over $\mathbb{F}_{p}$. We again denote the vectors by $P_{1}, \ldots, P_{N}$. Let $\mathcal{G}(S, D)$ be the matrix whose columns are the vectors $P_{1}, P_{2}, \ldots, P_{N}$. Then, $\mathcal{G}(S, D)$ is a $\kappa \times N$ matrix over $\mathbb{F}_{p}$ of rank $\kappa$. Define $C(S, D)$ to be the linear code with generator matrix $\mathcal{G}(S, D)$ so that $C$ is an $[N, \kappa]$-linear code over $\mathbb{F}_{p}$. In order to estimate the minimum distance of $C(S, D)$, we make use of Lemma 3.2. By (ii) of the lemma, if $C(S, D)$ has minimum distance $\delta$, there must be $N-\delta$ columns of $\mathcal{G}(S, D)$ of rank $\kappa-1$. Let $\mathcal{S}^{*}$ be the set of places represented by these columns. Then, there is a subgroup $G\left(\mathcal{S}^{*}, D\right)$ of $C \ell_{D}^{+}(A)$ containing $C \ell_{D}^{+}(A)^{p}$ and $\mathcal{S}^{*}$ such that $\left[C \ell_{D}^{+}(A): G\left(\mathcal{S}^{*}, D\right)\right]=p$. Let $L\left(\mathcal{S}^{*}, D\right)$ be the subfield of $F^{\prime}$ fixed by $G\left(\mathcal{S}^{*}, D\right)$. Then, $\left[L\left(\mathcal{S}^{*}, D\right): F\right]=p$ and 
by consideration of the Artin symbols, it is clear that $\infty$ and all the places in $\mathcal{S}^{*}$ split completely in $L\left(\mathcal{S}^{*}, D\right) / F$. If we assume further that all the places in $S$ are rational,

$$
p(N-\delta+1)+\epsilon \leq N\left(L\left(\mathcal{S}^{*}, D\right)\right) \leq N_{q}\left(g\left(L\left(\mathcal{S}^{*}, D\right)\right)\right),
$$

where

$$
\epsilon= \begin{cases}1 & \text { if } d=1 \\ 0 & \text { if } d>1\end{cases}
$$

In this way, knowledge of the upper bound of $\delta$ or $N_{q}(g)$ enables us to estimate the lower bounds of $N\left(L\left(\mathcal{S}^{*}, D\right)\right)$ or $\delta$, respectively. As the subsequent sections demonstrate, this construction provides us with some good codes and curves.

Next, let $C(S, D)^{\perp}$ be the dual code of $C(S, D)$. Then, $C(S, D)^{\perp}$ is an $[N, N-\kappa]$ linear code over $\mathbb{F}_{p}$ and

$$
C(S, D)^{\perp}=\left\{\left(c_{1}, c_{2}, \ldots, c_{N}\right) \in \mathbb{F}_{p}^{N}: \sum_{i=1}^{N} c_{i} P_{i}=0 \in V_{D}\right\} .
$$

The theorem below gives a lower bound for the minimum distance of $C(S, D)^{\perp}$.

Theorem 3.3. Let $S$ consist of all the rational places of $F$ different from $\infty$ and possibly $P$ when $\operatorname{deg} P=1$. Then, the code $C\left(S, P^{n}\right)^{\perp}$ has minimum distance $\delta^{\prime}$, where

$$
\delta^{\prime} \geq \begin{cases}n d+1-2 g(F) & \text { if } p \mid n, \\ (n-1) d+1-2 g(F) & \text { otherwise. }\end{cases}
$$

Proof. Let $\mathbf{c}$ be a codeword of $C\left(S, P^{n}\right)^{\perp}$ with weight $w(\mathbf{c})=w$. Without loss of generality, we may assume that the first $w$ coordinates of $\mathbf{c}$ are nonzero. Write $\mathbf{c}=\left(c_{1}, \ldots, c_{w}, 0, \ldots, 0\right)$. Since $\mathcal{G}\left(S, P^{n}\right)$ is a parity-check matrix of $C\left(S, P^{n}\right)^{\perp}$,

$$
\mathbf{c} \mathcal{G}\left(S, P^{n}\right)^{T}=\left(c_{1}, \ldots, c_{w}, 0, \ldots, 0\right)\left[P_{1} P_{2} \ldots P_{N}\right]=0,
$$

i.e.

$$
\sum_{i=1}^{w} c_{i} P_{i}=0 \in V_{D}
$$

This implies that there is a divisor $D^{\prime}$ of $F$ such that the divisor $X=\sum_{i=1}^{w} c_{i}\left(P_{i}-\infty\right)$ and $X^{\prime}=p D^{\prime}-p \operatorname{deg} D^{\prime} \infty$ are equivalent in the group $C \ell_{n P}^{+}(F)$. Furthermore, by the strong approximation theorem, it is easy to verify that $D^{\prime}$ can be chosen such that it is effective and $\operatorname{supp}\left(D^{\prime}\right) \cap\left\{\infty, P, P_{1}, \ldots, P_{N}\right\}=\emptyset$. We can therefore find an element $z \in F$ with

$$
X-X^{\prime}=\operatorname{div}(z)
$$

and $\nu_{P}(z-1) \geq n$. Consider the subfield $F_{0}=\mathbb{F}_{q}(z)$. We claim that the extension $F / F_{0}$ is separable. Suppose not. Then, $z \in F^{p}$, i.e. there is a $u \in F$ with $z=u^{p}$. This means that

$$
\operatorname{div}(z)=\operatorname{div}\left(u^{p}\right)=p \operatorname{div}(u) \equiv 0 \bmod p .
$$

Thus $X-X^{\prime} \equiv 0 \bmod p$ which shows that $p \mid c_{i}$ for all $1 \leq i \leq w$. This clearly contradicts our assumption that $\mathbf{c}$ is nonzero. Consequently, $F / F_{0}$ is separable. Since $\operatorname{div}(z)=\sum_{Q} \nu_{Q}(z) Q$, the ramification index of any place $Q$ in $\operatorname{supp}(\operatorname{div}(z))$ 
lying over the zero place of $z$ is just $|\nu|$ and the corresponding different exponents $d_{Q}$ for $Q$ lying over $z$ are as follows:

$$
\begin{aligned}
d_{P_{i}} & \geq c_{i}-1, i=1, \ldots, w \\
d_{Q} & \geq p \operatorname{deg} Q \text { for } Q \in \operatorname{supp}\left(D^{\prime}\right), \\
d_{\infty} & \geq\left|p \operatorname{deg} D^{\prime}-\sum_{i=1}^{w} c_{i}\right|-1 .
\end{aligned}
$$

Similarly, the ramification index of $P$ lying over the place $z-1$ is at least $n$, which makes the different exponent $d_{P}$ to be $d_{P} \geq n^{\prime}$, where

$$
n^{\prime}= \begin{cases}n & \text { if } p \mid n, \\ n-1 & \text { otherwise. }\end{cases}
$$

Applying the Hurwitz genus formula, we obtain

$$
\begin{aligned}
2 g(F)-2= & {\left[F: F_{0}\right](-2)+\sum_{Q \in \mathbf{P}_{F}} d_{Q} \operatorname{deg} Q } \\
\geq & -2\left|p \operatorname{deg} D^{\prime}-\sum_{i=1}^{w} c_{i}\right|+\sum_{i=1}^{w}\left(c_{i}-1\right)+p \operatorname{deg} D^{\prime} \\
& +\left|p \operatorname{deg} D^{\prime}-\sum_{i=1}^{w} c_{i}\right|-1+n^{\prime} d \\
\geq & -w+n^{\prime} d-1 .
\end{aligned}
$$

As a result, $w \geq n^{\prime} d+1-2 g$, which in turn implies that the minimum distance of $C\left(S, P^{n}\right)^{\perp}$ must satisfy

$$
\delta^{\prime} \geq n^{\prime} d+1-2 g
$$

\section{EXPLiCIT EXAMPLES OF CODES}

We now give some examples of codes constructed as in the preceding section and their parameters. For the examples considered here, let $n=2$, i.e. $D=2 P$.

Theorem 4.1. Let $F$ be a global function field with genus $g$ and $N(F)$ rational places. Let

$$
\epsilon= \begin{cases}1 & \text { if } d=1 \\ 0 & \text { if } d>1 .\end{cases}
$$

For $N \leq N(F)-1-\epsilon$, let $S \subseteq \mathbf{P}_{F}-\{P, \infty\}$ consist of $N$ distinct rational places. Assume that $S$ generates $C \ell_{D}^{+}(A)$ and that $N_{q}(p g-p+1) \leq N_{q}(p g+(d-1)(p-1))$. Then, the code $C\left(S, P^{2}\right)$ constructed as described above is an $[N, \kappa, \delta]$-code over $\mathbb{F}_{p}$, where $\kappa \geq$ rd and $\delta$ satisfies

$$
\delta \geq N+1-\left\lfloor\frac{N_{q}(p g+(d-1)(p-1))-\epsilon}{p}\right\rfloor .
$$

Proof. The length $N$ is trivial. So let $\delta$ be the minimum distance of $C\left(S, P^{2}\right)$. By Lemma 3.2, there must exist $N-\delta$ columns of $\mathcal{G}\left(S, P^{2}\right)$ of rank $\kappa-1$. Let $S^{\prime}$ be the set of places represented by these $N-\delta$ columns. Construct the field $L$ of $F$ such that $[L: F]=p$ and all the places in $S^{\prime}$ as well as $\infty$ split completely in $L / F$. As such, $L$ has at least $p(N-\delta+1)$ rational places. It remains to calculate the 
genus of $L$. Observe that $P$ can be either unramified or totally ramified in $L / F$. In the former case, the Hurwitz genus formula gives

$$
g(L)=1+p(g-1)=p g-p+1 .
$$

In case $P$ is totally ramified in $L / F$, since the conductor of $P$ in $L / F \leq 2$, the genus of $L$ is, according to [15, Theorem 2.3.6], given by

$$
g(L)=1-d+p(g-1+d)=p g+(d-1)(p-1) .
$$

Since

$$
N_{q}(p g-p+1) \leq N_{q}(p g+(d-1)(p-1))
$$

by our assumption, the maximum number of rational places in $L / F$ is $N_{q}(p g+(d-1)(p-1))$. Consequently,

$$
p(N-\delta+1) \leq N_{q}(p g+(d-1)(p-1))-\epsilon
$$

and our desired bound on $\delta$ follows. Finally as $S$ generates $C \ell_{D}^{+}(A), \kappa \geq r d$.

The next corollary looks at a special case of Theorem 4.1.

Corollary 4.2. Let $F$ be the rational function field $F=\mathbb{F}_{q}(x)$. Suppose that all other notation is as in Theorem 4.1. Then, the code $C\left(S, P^{2}\right)$ is an $[N, \kappa, \delta]$-linear code over $\mathbb{F}_{p}$, where

$$
\begin{aligned}
N & \leq q-\epsilon, \\
\kappa & =r d, \\
\delta & \geq N+1-\left\lfloor\frac{N_{q}((p-1)(d-1))-\epsilon}{p}\right\rfloor .
\end{aligned}
$$

In particular, for $d=1$ and $N=q-i, 1 \leq i \leq p$, the code $C\left(S, P^{2}\right)$ is optimal.

Proof. Since $F$ is the rational function field, $g=0$ and $N(F)=q+1$. Further, $C \ell_{P^{2}}^{+}(A)=\left(A / P^{2}\right)^{*}$, which gives $\kappa=r d$. Since any intermediate field of $F^{\prime} / F$ must be ramified, the first assertion follows from Theorem 4.1 Now, let $d=1$ and $N=q-i, 1 \leq i \leq p$. Since $g(L)=0, L$ is also a rational function field. Therefore, $C\left(S, P^{2}\right)$ is a $[q-i, r, q-i+1-q / p]=\left[p^{r}-i, r, p^{r-1}(p-1)-i+1\right]$-linear code over $\mathbb{F}_{p}$. Since

$$
\begin{aligned}
\sum_{j=0}^{r-1}\left\lceil\frac{p^{r-1}(p-1)-i+1}{p^{j}}\right\rceil & =\sum_{j=0}^{r-1} p^{j}(p-1)-i+1 \\
& =p^{r}-i \\
& =N,
\end{aligned}
$$

the Griesmer bound implies that $C\left(S, P^{2}\right)$ is optimal.

Example 1. (1) Let $\delta=2$ and $q=64$ in Corollary 4.2 Since $N_{64}(1)=81$, we obtain the binary $[64,12,25]$ and $[63,12,24]$ linear codes, which are the best known (see [4]).

(2) Let $\delta=2$ and $q=256$ in Corollary 4.2. Since $N_{256}(1)=289$, we obtain the binary $[256,16,113]$ and $[255,16,112]$ linear codes, which are the best known (see [4]). 
Theorem 4.3. Let $F=\mathbb{F}_{q}(x)$ be the rational function field. Suppose that $N=$ $\frac{q^{p}-q}{p}$. Let $S \subseteq \mathbf{P}_{F}$ consist of $N$ distinct places of $F$ of degree $p$ and let $P$ be a place of $S$ with degree $\operatorname{deg} P=d \neq p$. Then, the code $C\left(S, P^{2}\right)$ is an $[N, r d, \delta]$-linear code over $\mathbb{F}_{p}$, where

$$
\delta \geq \begin{cases}N+\left\lceil\frac{p q+p-N_{q} p((d-1)(p-1))}{p^{2}}\right\rceil & \text { if } d>1 \\ \frac{(p-1) q^{p}}{p^{2}} & \text { if } d=1 .\end{cases}
$$

Proof. Let $\delta$ be the minimum distance of $C\left(S, P^{2}\right)$. Lemma 3.2 implies that there are $N-\delta$ columns of the generator matrix $\mathcal{G}\left(S, P^{2}\right)$ of rank $\kappa-1$ with $\kappa$ being the dimension of $C\left(S, P^{2}\right)$. Denote by $S^{*}$ the set of all places represented by these columns. Construct the field $L=L\left(\mathcal{S}^{*}, P^{2}\right)$. Further, assume that $\delta^{\prime}$ rational places split in $L / F$. Then $L$ has $p(N-\delta)+q+1-\delta^{\prime}+\epsilon$ places of degree $p$ and $p \delta^{\prime}+\epsilon$ rational places, where

$$
\epsilon= \begin{cases}1 & \text { if } d=1 \\ 0 & \text { if } d>1\end{cases}
$$

Let $L^{\prime}$ be the constant field extension $L^{\prime}=L \mathbb{F}_{q^{p}}$. Since every place of $L$ of degree $p$ splits into $p$ rational places in $L^{\prime}$ and every rational place of $L$ remains rational in $L^{\prime}, L^{\prime}$ has at least

$N\left(L^{\prime}\right)=p^{2}(N-\delta)+p\left(q+1-\delta^{\prime}-\epsilon\right)+p \delta^{\prime}+\epsilon=p^{2}(N-\delta)+p(q+1)+\epsilon(p-1)$

rational places. Since $P$ is the only ramified place in $L$ with conductor $\leq 2$, the Hurwitz genus formula yields

$$
g\left(L^{\prime}\right)=g(L)=(d-1)(p-1) .
$$

Thus we have $N\left(L^{\prime}\right) \leq N_{q^{p}}((d-1)(p-1))$, which yields our desired assertions.

Example 2. Let $p=2$ and $d=1$ in the preceding theorem. We obtain binary linear codes with parameters $\left[2^{r-1}\left(2^{r}-1\right), r, 2^{2 r-2}\right]$. By the Griesmer bound (see [14, Theorem 5.7.4]), these codes are optimal.

\section{Global Function fields from Codes}

In the previous section, we made use of upper bounds on $N_{q}(g)$ to estimate the minimum distance of $C\left(S, P^{2}\right)$. In this present section, we will illustrate how the construction described in Section 3 allows us to obtain the fields $L\left(\mathcal{S}^{*}, D\right)$ having a large number of rational places using known upper bounds on minimum distances.

More precisely, we first choose a suitable base field $F / \mathbb{F}_{q}$ with $\operatorname{char}\left(\mathbb{F}_{q}\right)=p$, a rational place $\infty$ of $F$ and a set $S$ consisting of $N$ other rational places of $F$. Fix a place $P$ of degree $d$ for some positive integer $d$ and form the generator matrix $\mathcal{G}\left(S, P^{2}\right)$ and the code $C\left(S, P^{2}\right)$ as described in Section 3. From some upper bounds on the minimum distance of $C\left(S, P^{2}\right)$ as well as the conclusions of Lemma 3.2 we can find a subset $\mathcal{S}^{*}$ of $S$ and a field $L\left(\mathcal{S}^{*}, P^{2}\right)$ in which all places in $\mathcal{S}^{*}$ and $\infty$ split completely in the extension $L\left(\mathcal{S}^{*}, P^{2}\right) / F$. Notice here that we have made use of known results in coding theory to help us construct a large linearly dependent subset $\mathcal{S}^{*}$ of $S$ meeting our conditions.

Example 3. $G\left(L / \mathbb{F}_{2}\right)=52, N\left(L / \mathbb{F}_{2}\right) \geq 36$. Consider the function field $F / \mathbb{F}_{2}$ in which $g(F)=19$ and $N(F)=20$. Such an $F$ exists by [22]. Fix a rational place $\infty$ and let $S$ contain all the remaining rational places of $F$. By [15, Lemma 1.6.13], we can find a place $P$ of $F$ with degree 15 . Then the code $C\left(S, P^{2}\right)$ is a binary 
code with length 19 and dimension at least 15. Consider the parity-check matrix $\mathcal{H}\left(S, P^{2}\right)$ of $C\left(S, P^{2}\right)$. Clearly, $\mathcal{H}\left(S, P^{2}\right)$ is a $t \times 19$ matrix over $\mathbb{F}_{2}$ with $t \leq 4$. As there are at most 15 distinct nonzero $t$-tuples, we conclude from Lemma 3.2 (i) that $C\left(S, P^{2}\right)$ has minimum distance at most 2. Consequently, by Lemma 3.2 (ii), there are 17 columns of $\mathcal{G}\left(S, P^{2}\right)$ of rank at most 14 . Letting $\mathcal{S}^{*}$ consist of these 17 columns, construct the field $L=L\left(\mathcal{S}^{*}, P^{2}\right)$. From (3),

$$
N(L) \geq 2(18)=36 \text {. }
$$

By the proof of Theorem 4.1, the genus of $L$ is

$$
g(L)=2(19-1)=36
$$

or

$$
g(L)=2(19)+14=52 .
$$

Since $N_{2}(36)=31<36$ according to the table in [22], we conclude that $g(L)=52$. The Oesterle bound for $N_{2}(52)$ is 42 . Note that this example improves the bound of $N_{2}(52) \geq 34$ given in [15, Table 4.5.2].

Example 4. $g\left(L / \mathbb{F}_{128}\right)=13, N\left(L / \mathbb{F}_{128}\right) \geq 308$. Consider the function field $F / \mathbb{F}_{128}$ in which $g(F)=4$ and $N(F)=215$. Such an $F$ exists by [22]. Fix a rational place $\infty$ and let $S$ consist of all the remaining rational places of $F$. By [15, Lemma 1.6.3], we can find a place $P$ of $F$ with degree 6 . Then the code $C\left(S, P^{2}\right)$ is a binary code with length 214 and dimension at least 42. Now, by Brouwer's table [4, the lower bound on the minimum distance of a [214,42]-code is 61 while the upper bound is 82. Let $\delta$ be the minimum distance of $C\left(S, P^{2}\right)$. Thus, $\delta \leq 82$. By Lemma 3.2 (ii), there are $214-\delta$ columns in the matrix $\mathcal{G}\left(S, P^{2}\right)$ with rank at most 41 . With $\mathcal{S}^{*}$ containing these $214-\delta$ columns, construct the field $L=L\left(\mathcal{S}^{*}, P^{2}\right)$. According to 3.

$$
N(L) \geq 2(215-\delta)=430-2 \delta \geq 266 .
$$

From the proof of Theorem 4.1 the genus of $L$ is given by

$$
g(L)=2(4-1)=6
$$

or

$$
g(L)=2(4)+5=13 .
$$

Since $N_{128}(6) \leq 258$ according to the table [22, we conclude that $g(L)=13$. Now, since the best known code of length 214 and dimension 42 has minimum distance 61 , we may assume that $\delta \leq 61$. In this case, $N(L) \geq 308$. By the Oesterle bound, $N_{128}(13)=428$. Since $\lceil 428 / \sqrt{2}\rceil=299$, the field $L$ constructed here has more rational places than the criteria for the lower entry of $N_{128}(13)$ in [22].

Remark 5.1. In Example 4 notice that for $61 \leq \delta \leq 65, L$ has sufficient rational places that meet the criteria for the lower entry of $N_{128}(13)$ in [22]. In this case, both the code $C\left(S, P^{2}\right)$ and the field $L$ are better than the existing code and field with the same parameters. For $66 \leq \delta \leq 82$, the code $C\left(S, P^{2}\right)$ has a minimum distance that improves the current lower bound. 


\section{Cyclotomic Function fields}

The codes constructed so far are not explicit in the sense that the generator matrix cannot be written down explicitly. In this section, we will apply the construction in Section 3 to a special family of narrow ray class fields.

Let $F$ be the rational function field $F=\mathbb{F}_{q}(x)$, where $x$ is an indeterminate, and let $\infty$ denote the pole of $x$. The narrow ray class field $F^{\prime}=F^{D}(\infty)$ is better known as the cyclotomic function field with modulus $D[10$. In this case, $P$ is simply a monic irreducible polynomial over $\mathbb{F}_{q}$ of degree $d$ and $A=\mathbb{F}_{q}[x]$. Since $H_{A}=F$, it follows that $\operatorname{Gal}\left(F^{\prime} / F\right)=\left(\mathbb{F}_{q}[x] / P^{n}\right)^{*}$.

Our goal is to construct subfields $L$ of $F^{\prime}$ in which many rational places of $F$ split completely in $L / F$. Equivalently, we wish to construct subgroups $\operatorname{Gal}\left(F^{\prime} / L\right)$ of $\left(A / P^{n}\right)^{*}$ which contain many linear polynomials. Looking at Theorem 2.6, the genus of $L$ can be easily computed once we know the orders of certain groups generated by the linear polynomials. Unfortunately, computing these orders may not be so straightforward, especially when $q$ is large. In what follows, we will show that the code construction introduced in this paper facilitates this process in certain cases. By employing mathematical software packages such as Mathematica to carry out our computations, we are therefore able to construct global function fields having more rational places than existing ones with the same genera.

The proposition below will be needed. For any positive integer $n$, denote by $n_{p}^{*}$ the smallest integer $l$ such that $n \leq p^{l}$ and $n_{p}=p^{n_{p}^{*}}$.

Proposition 6.1. Suppose that there is an integer $i$ such that $P_{k} \in\left\langle P_{1}, \ldots, P_{k-1}\right\rangle_{i}$ but $P_{k} \notin\left\langle P_{1}, \ldots, P_{k-1}\right\rangle_{i+1}$. Then

$$
\left|\left\langle P_{1}, \ldots, P_{k}\right\rangle_{n}\right|=\left|\left\langle P_{1}, \ldots, P_{k-1}\right\rangle_{n}\right|(n / i)_{p}
$$

Proof. If $n \leq i$, then the result is obvious. So we will assume that $n>i$. For

an integer $j$, let $G_{j}=\left\langle P_{1}, P_{2}, \ldots, P_{k-1}\right\rangle_{j}$. It is clear that $\left|\left\langle P_{k}\right\rangle_{n}\right|=a n_{p}$, where $a=\left|\left\langle P_{k}\right\rangle_{1}\right|$. Since $P_{k} \in G_{1}$, it follows that $\left|G_{n} \cap\left\langle P_{k}\right\rangle_{n}\right|=a p^{l}$ for some integer $l$. We claim that $l=n_{p}^{*}-(n / i)_{p}^{*}$. Indeed, by our assumption, $P_{k} \in G_{i}$ and thus, $P_{k}^{n_{p}} \in G_{n}$ as $i n_{p}>n$. So $l \geq n_{p}^{*}-(n / i)_{p}^{*}$. If $P_{k}^{p^{j}} \in G_{n}$ for $j<(n / i)_{p}^{*}$, we will have $P_{k} \in G_{\left\lfloor n / p^{j}\right\rfloor}>i$. Thus, $l \leq n_{p}^{*}-(n / i)_{p}^{*}$ and equality holds. Consequently,

$$
\left|\left\langle P_{1}, P_{2}, \ldots, P_{k}\right\rangle_{n}\right|=\left|H_{n}\right|\left|\left\langle P_{k}\right\rangle_{n}\right| / p^{l}=\left|H_{n}\right|(n / i)_{p} .
$$

For the remainder of this section, we refer to the notation in Theorem 2.6. For $i=1, \ldots, n$, we further denote by $\Omega\left(P^{i}\right)$ for the order $\left|\left(A / P^{i}\right)^{*} / G_{i P}\right|$.

First of all, we let $q=p$ and concentrate on cyclotomic function fields over the prime field $\mathbb{F}_{p}$. In this case, $F$ has $p$ rational places apart from $\infty$.

Definition 6.2. A finite set $\mathcal{S}^{*} \subseteq \mathbf{P}_{F}-\{P, \infty\}$ is said to be independent $\bmod$ $P^{n}$ if it is $\mathbb{F}_{p}$-linearly independent in the vector space $V_{P^{n}}$ (recall the definition of $V_{P^{n}}$ from Section 3).

Let $S$ consist of all the rational places of $F$ other than $\infty$ and possibly $P$, when $\operatorname{deg} P=1$. As such, $|S|=p-\epsilon$, where

$$
\epsilon= \begin{cases}1 & \text { if } d=1 \\ 0 & \text { otherwise }\end{cases}
$$

Consider the code $C\left(S, P^{n}\right)$. Then, $C\left(S, P^{n}\right)$ is a $[p-\epsilon, \kappa \leq d(n-1-\lfloor(n-1) / p\rfloor)]$ code over $\mathbb{F}_{p}$. First, consider $n<\lfloor(p-\epsilon) / d\rfloor+1<p$. Now by Theorem 3.3 , 
$C\left(S, P^{n}\right)^{\perp}$ has minimum distance at least $(n-1) d+1$. Applying Lemma 3.2 (i), and since the number of linearly independent vectors cannot exceed the dimension $\kappa \leq d(n-1)$, we can conclude that $C\left(S, P^{n}\right)^{\perp}$ has minimum distance exactly $(n-1) d+1$. Consequently, any subset of $\leq d(n-1)$ rational places of $F$ must be independent $\bmod P^{n}$ and $\kappa=d(n-1)$. On the other hand, for $n \geq\lfloor(p-\epsilon) / d\rfloor+1$, it is clear that the generator matrix $\mathcal{G}\left(S, P^{n}\right)$ is a $(p-\epsilon) \times(p-\epsilon)$ invertible matrix; i.e., all $p-\epsilon$ rational places are independent $\bmod P^{n}$. Together with Proposition 6.1. we can easily prove the following lemma.

Lemma 6.3. Let $\mathcal{S}^{*} \subset \mathbf{P}_{F}-\{\infty, P\}$ consist of $k$ rational places of $F$, where $k \leq p-\epsilon$. Write $k=a d+b$, where $0 \leq b<d$. Then for any positive integer $n>1$, we have

$$
\Omega\left(P^{n}\right)=\omega \frac{p^{n-1}}{(n /(a+1))_{p}^{b} \prod_{i=1}^{a}(n / i)_{p}^{d}},
$$

where $\omega=\Omega(P)$.

Proof. Let $\mathcal{S}^{*}=\left\{P_{1}, P_{2}, \ldots, P_{k}\right\}$. For $i=1, \ldots, d$, it is clear from Proposition 6.1 that $\left|\left\langle P_{i}\right\rangle_{n}\right|=\omega_{i} n_{p}$, where $\omega_{i}=\left|\left\langle P_{i}\right\rangle_{1}\right|$. Thus,

$$
\left|\left\langle P_{1}, \ldots, P_{d}\right\rangle_{n}\right|=\omega^{\prime} n_{p}^{d},
$$

where $\omega^{\prime}=\left|\left\langle P_{1}, \ldots, P_{d}\right\rangle_{1}\right|$. From our above discussion, $P_{d+1} \in\left\langle P_{1}, \ldots, P_{d}\right\rangle_{2}$ but $P_{d+1} \notin\left\langle P_{1}, \ldots, P_{d}\right\rangle_{3}$. Applying Proposition 6.1 yields $\left|\left\langle P_{1}, \ldots, P_{d+1}\right\rangle_{n}\right|=$ $\omega^{\prime}\left|\left\langle P_{1}, \ldots, P_{d}\right\rangle_{n}\right|(n / 2)_{p}=\omega^{\prime} n_{p}^{d}(n / 2)_{p}$. Continuing in this way for $i=d+2, \ldots, k$ and recalling that $\Phi\left(P^{n}\right)=\left(p^{d}-1\right) p^{d(n-1)}$, we obtain

$$
\left|\left\langle P_{1}, \ldots, P_{k}\right\rangle_{n}\right|=\omega^{\prime}(n /(a+1))_{p}^{b} \prod_{i=1}^{b}(n / i)_{p}^{d} .
$$

Consequently,

$$
\Omega\left(P^{n}\right)=\omega \frac{p^{n-1}}{(n /(a+1))_{p}^{b} \prod_{i=1}^{a}(n / i)_{p}^{d}},
$$

where $\omega=\left(p^{d}-1\right) / \omega^{\prime}=\Omega(P)$.

With these computations, the genus and number of rational places of the field $L$ constructed in Theorem 2.6 can be written down explicitly.

Corollary 6.4. Let $\mathcal{S}^{*} \subset \mathbf{P}_{F}-\{\infty, P\}$ consist of $k$ rational places of $F$, where $k \leq p-\epsilon$. Write $k=a d+b$, where $0 \leq b<d$. Then the subfield $L$ of the cyclotomic function field $F\left(\Lambda_{P^{n}}\right) / F$ constructed as the fixed field of $G_{P^{n}}$ has genus

$$
2 g(L)-2=\Omega\left(P^{n}\right)(d n-2)-d\left(1+\sum_{i=1}^{n-1} \Omega\left(P^{i}\right)\right),
$$

where $\Omega\left(P^{j}\right)=\Omega(P) \frac{p^{d(j-1)}}{(j /(a+1))_{p}^{b} \prod_{i=1}^{a}(j / i)_{p}^{d}}$ for $n \geq 2$. Further, L has exactly

$$
N(L)=(k+1) \Omega\left(P^{n}\right)+\epsilon
$$

rational places.

Example 5. Put $d=1$ in the above corollary. Then

$$
g(L)=\frac{p^{n-1}}{2 \prod_{i=1}^{k}(n / i)_{p}}(n-2)-\sum_{j=2}^{n-1} \frac{p^{j-1}}{2 \prod_{i=1}^{k}(j / i)_{p}}
$$


and

$$
N(L)=\frac{p^{n-1}}{\prod_{i=1}^{k}(n / i)_{p}}(k+1)+1 .
$$

Next we return to the case $q=p^{r}$ but we let $P=x$. Let $\alpha$ be a fixed primitive element of $\mathbb{F}_{q}^{*}$. We denote by $P_{i}$ the zero of $x-\alpha^{i}, i=0, \ldots, q-2$. Let $S=$ $\left\{P_{0}, P_{1}, \ldots, P_{N-1}\right\}$, where $N=q-1$ and construct the code $C\left(S, x^{n}\right)$. Write the generator matrix of $C\left(S, x^{n}\right)$ as

$$
\mathcal{G}\left(S, x^{n}\right)=\left[P_{0} P_{1} \ldots P_{N-1}\right] .
$$

Let us recall Newton's formulas involving the sums of powers of roots of a polynomial.

Lemma 6.5 (Newton's formula). Let $f(x)=\sum_{i=0}^{l} \beta_{i} x^{i}$ be a polynomial over $\mathbb{F}_{p}$ with reciprocal roots $\alpha_{1}, \alpha_{2}, \ldots, \alpha_{l}$, i.e.

$$
f(x)=\prod_{i=1}^{l}\left(1-\alpha_{i} x\right) .
$$

For any integer $u$, let $y_{u}=\sum_{i=1}^{l} \alpha_{i}^{u}$. Then

$$
s \beta_{s}+\sum_{i=1}^{s} \beta_{i} y_{s-i}=0
$$

for any positive integer $s$.

Proposition 6.6. The code $C\left(S, x^{n}\right)^{\perp}$ is a narrow-sense $\mathrm{BCH}$ code with designed distance $\delta_{0}=n$.

Proof. Let $\mathbf{c}=\left(c_{0}, c_{1}, \ldots, c_{N-1}\right) \in C\left(S, x^{n}\right)^{\perp}$. We need to show that $\alpha^{s}$ is a root of $c(x)=\sum_{i=0}^{N-1} c_{i} x^{i}$ for all $s=1,2, \ldots, n-1$. Since $\mathcal{G}\left(S, x^{n}\right)$ is a parity-check matrix of $C\left(S, x^{n}\right)^{\perp}$, we have

$$
\sum_{i=0}^{N-1} c_{i} P_{i}=0
$$

By identifying $c_{i} \in \mathbb{F}_{p}$ with the corresponding integer $c_{i} \in \mathbb{Z}$, it follows that there exists a polynomial $f(x) \in\left(\mathbb{F}_{q}[x] /\left(x^{n}\right)\right)^{*}$ such that

$$
\prod_{i=0}^{N-1}\left(x-\alpha^{i}\right)^{c_{i}} \equiv f(x)^{p} \equiv f\left(x^{p}\right) \bmod \left(p, x^{n}\right) .
$$

Factoring out the constants, the above is equivalent to

$$
\prod_{i=0}^{N-1}\left(1-\alpha^{i} x\right)^{c_{i}} \equiv f^{\prime}\left(x^{p}\right) \bmod \left(p, x^{n}\right)
$$

for some $f^{\prime}(x)$. Now write $\prod_{i=0}^{N-1}\left(1-\alpha^{i} x\right)^{c_{i}}=\sum_{j=0}^{\infty} \beta_{j} x^{j}$. Clearly, $\beta_{0}=1$ and $\beta_{j} \equiv 0 \bmod p$ for all $j=1, \ldots, n-1$ and $\operatorname{gcd}(j, p)=1$. We claim that for integers $s=1, \ldots, n-1$,

$$
c\left(\alpha^{s}\right)=\sum_{i=0}^{N-1} c_{i} \alpha^{i s}=0 .
$$

We prove this by induction on $s<n$. By Newton's formulas,

$$
\beta_{1} \equiv-\beta_{0} c(\alpha) \equiv-c(\alpha) \equiv 0 \bmod p .
$$


The result is thus true for $s=1$. So assume that the result holds for all positive integers $\leq s-1<n$. By Newton's formulas again,

$$
s \beta_{s}=-\sum_{j=1}^{s} \beta_{j} c\left(\alpha^{s-j}\right) \bmod p .
$$

By our induction hypothesis, this becomes $c\left(\alpha^{s}\right) \equiv-s \beta_{s} \bmod p$. But $s \beta_{s} \equiv 0 \bmod p$ for all $s<n$. Hence our claim is shown. Consequently, $C\left(S, x^{n}\right)^{\perp}$ is a narrow-sense $\mathrm{BCH}$ code with designed distance at least $n$. By the arguments just discussed, it is clear that if $c(x)$ is the lcm of all the minimal polynomials of $\alpha^{j}$ for $j=1, \ldots, n-1$, then the codeword $\mathbf{c}$ corresponding to $c(x)$ is a codeword in $C\left(S, x^{n}\right)^{\perp}$. From the definition of a narrow-sense BCH code, this shows that the designed distance of $C\left(S, x^{n}\right)^{\perp}$ is exactly $n$.

Corollary 6.7. $C\left(S, x^{n}\right)$ is a cyclic code with generator polynomial $g(x)=$ $x^{N} h(1 / x)$, where

$$
h(x)=\operatorname{lcm}\left(m_{1}(x), \ldots, m_{n-1}(x)\right)
$$

and for $1 \leq i \leq n-1, m_{i}(x)$ is the minimal polynomial of $\alpha^{i}$ over $\mathbb{F}_{p}$.

Proof. This follows immediately from standard results of cyclic codes [14.

Corollary 6.8. The code $C\left(S, x^{n}\right)$ has dimension $\kappa=r(n-1-\lfloor(n-1) / p\rfloor)$ if $n \leq p^{\lceil r / 2\rceil}$.

Proof. It is well known in the study of BCH codes that for $n \leq p^{\lceil r / 2\rceil}, C\left(S, x^{n}\right)^{\perp}$ has dimension $q-1-r(n-1-\lfloor(n-1) / p\rfloor)$. Hence, the dimension of $C\left(S, x^{n}\right)$ is $r(n-1-r\lfloor(n-1) / p\rfloor)$.

Remark 6.9. The rank of $C\left(S, x^{n}\right)$ was first shown by Lauter in [13] using generalized Witt vectors. However, our proof follows that given in [1.

For the codes discussed in this section, we have shown that their structures are explicitly known. As such, we can exploit mathematical software packages such as Mathematica or Magma to look for subsets $\mathcal{S}^{*}$ of $S$ of different ranks, and then construct the associated fields as discussed in Section 3. Lemma 3.2 suggests how we can search for good subsets.

More specifically, we define recursively the codes $C_{0}, C_{1}, \ldots$ and their respective fields $L_{0}, L_{1}, \ldots$ as follows. Let $C_{0}=C\left(S, x^{n}\right), S_{0}=S, \delta_{0}=d\left(C_{0}\right)$, and $\mathbf{c}_{0} \in C_{0}$ with weight $w\left(\mathbf{c}_{0}\right)=\delta_{0}$. Construct $L_{0}$ by the method following Lemma 3.2 with $\mathcal{S}^{*}=S-\operatorname{supp}\left(\mathbf{c}_{0}\right)$ as the generating set. Now, assume that $C_{i}, S_{i}, \delta_{i}, \mathbf{c}_{i}$ and $L_{i}$ have been constructed. Let $S_{i+1}=S_{i}-\operatorname{supp}\left(\mathbf{c}_{i}\right), C_{i+1}$ be the residual code of $C_{i}$ at $\mathbf{c}_{i}$, $\delta_{i+1}=d\left(C_{i+1}\right), \mathbf{c}_{i+1} \in C_{i+1}$ with $w\left(\mathbf{c}_{i+1}\right)=\delta_{i+1}$ and $L_{i+1}$ as the field constructed with $\mathcal{S}^{*}=S_{i+1}-\operatorname{supp}\left(\mathbf{c}_{i+1}\right)$ as the generating set.

Proposition 6.10. Suppose that $C\left(S, x^{n}\right)$ has dimension $\left.n-1-\lfloor(n-1) / p\rfloor\right)$. With $C_{i}, S_{i}, \delta_{i}, \mathbf{c}_{i}$ and $L_{i}$ as above, the following are true.

(i) For $i=0, \ldots, r-1,\left[L_{i}: F\right]=p^{i+1}$.

(ii) All places in $S_{i+1}$ split completely in $L_{i} / F$. In particular, $L_{i}$ has $\left(\left|S_{i+1}\right|+1\right) p^{i}+1=\left(q-\sum_{i=0}^{i} \delta_{i}\right) p^{i+1}+1$ rational places.

(iii) The genus of $L_{i}$ is given by

$$
g\left(L_{i}\right) \leq\left(p^{i}-1\right)(n-2) / 2
$$

and equality holds if the places in $S_{i+1}$ generate $C\left(S, x^{n-1}\right)$. 
TABLE 1. Improved lower bounds on $N_{q}(g)$

\begin{tabular}{|c|l|l|l|l|l|l|l|}
\hline$q$ & $n$ & $i$ & $\left(\delta_{0}, \ldots, \delta_{i}\right)$ & $\left(\kappa_{n-1}, \kappa_{n-2}, \ldots\right)$ & $g$ & $N(L)$ & $N_{q}(g)$ \\
\hline 64 & 6 & 2 & $(16,8,8)$ & $(12, \ldots)$ & 14 & 257 & $241--284$ \\
\hline 64 & 8 & 2 & $(14,7,6)$ & $(18, \ldots)$ & 21 & 297 & $281--396$ \\
\hline 64 & 8 & 3 & $(14,7,6,5)$ & $(17,17,12, \ldots)$ & 44 & 513 & 695 \\
\hline 128 & 6 & 3 & $(48,24,14,7)$ & $(13,13,7, \ldots)$ & 29 & 561 & 785 \\
\hline 27 & 6 & 2 & $(9,3,3)$ & $(8,6, \ldots)$ & 51 & 361 & 423 \\
\hline 27 & 6 & 2 & $(9,3,1)$ & $(9, \ldots)$ & 52 & 379 & 430 \\
\hline 81 & 3 & 2 & $(48,16,6)$ & $(4)$ & 13 & 298 & $256-312$ \\
\hline 81 & 8 & 1 & $(30,10)$ & $(15,15,12, \ldots)$ & 22 & 370 & 478 \\
\hline 81 & 8 & 1 & $(30,10)$ & $(16, \ldots)$ & 24 & 370 & 514 \\
\hline 81 & 3 & 3 & $(48,16,6,2)$ & $(3)$ & 39 & 730 & $646-768$ \\
\hline 81 & 6 & 2 & $(30,15,8)$ & $(11,8, \ldots)$ & 51 & 757 & 923 \\
\hline 81 & 6 & 2 & $(30,10,13)$ & $(12, \ldots)$ & 52 & 757 & 936 \\
\hline
\end{tabular}

Proof. All the assertions can be proven by induction on $i$. As for the genus, we apply the genus formula given in Theorem 2.6.

With this procedure, we use Mathematica to determine the sequences $\left(\delta_{0}, \delta_{1}, \ldots\right)$ and their respective sets $\left(S_{0}, S_{1}, \ldots\right)$ for $q=27,32,64,81$ and 128 . The following new function fields are obtained. As in 22], we restrict our search for fields with genus at most 50. In addition, we give values for $N_{27}(51), N_{27}(52), N_{81}(51)$ and $N_{81}(52)$. Note that to compute the genus of $L_{i}$, we need to know the rank of the places in $S_{i+1}$ with respect to the codes $C\left(S, x^{j}\right), j<n$. Clearly, if the places in $S_{i+1}$ generate $C\left(S, x^{j}\right)$, they generate $C\left(S, x^{j^{\prime}}\right)$ for all $j^{\prime}<j$. In Table $1, \kappa_{j}$ refers to the rank $S_{i+1}$ with respect to the code $C\left(S, x^{j}\right)$. In the last column, we give the range of $N_{q}(g)$ taken from 22 . In the case where no entry is entered in that table, we provide the Oesterle bound for comparison.

\section{ACKNOWLEDGMENT}

The authors are grateful to the anonymous referee for his/her careful reading of the previous version of the paper and invaluable suggestions and comments which greatly improve the paper.

\section{REFERENCES}

[1] R. Auer, Ray class fields of global function fields with many rational places, Dissertation, University of Oldenburg, 1999.

[2] R. Auer, Curves over finite fields with many rational points obtained by ray class field extensions, Algorithmic Number Theory (W. Bosma, ed.), Lecture Notes in Computer Science, Vol. 1838, pp. 127-134, Springer, Berlin, 2000. MR.1850602 (2002h:11053)

[3] R. Auer, Ray class fields of global function fields with many rational places, Acta Arith. 95, 97-122 (2000). MR1785410(2002e:11162)

[4] A. E. Brouwer, Bounds on the Minimum Distance of Linear Codes, Website: http://www.win.tue.nl/ aeb/voorlincod.html

[5] C.S. Ding, H. Niederreiter, and C.P. Xing, Some new codes from algebraic curves, IEEE Trans. Inform. Theory 46, 2638-2642 (2000). MR1806824 (2001j:94048)

[6] N. D. Elkies, Excellent codes from modular curves, in STOC'01: Proceedings of the 33rd Annual ACM Symposium on Theory of Computing, Hersonissos, Crete, 200-208(2001). $\operatorname{MR} 2120316$ 
[7] V.D. Goppa, Codes on algebraic curves (Russian), Dokl. Akad. Nauk SSSR 259, 1289-1290 (1981). MR628795 (82k:94017)

[8] V.D. Goppa, Algebraic-geometric codes (Russian), Izv. Akad. Nauk SSSR Ser. Mat. 46, 762-781 (1982). MR670165 (84g:94011)

[9] V.D. Goppa, Geometry and Codes, Kluwer, Dordrecht, 1988. MR1029027(91a:14013)

[10] D.R. Hayes, Explicit class field theory for rational function fields, Trans. Amer. Math. Soc. 189, 77-91 (1974). MR0330106 (48:8444)

[11] D.R. Hayes, Explicit class field theory in global function fields, Studies in Algebra and Number Theory, Advances in Math. Supp. Studies, Vol. 6, pp. 173-217, Academic Press, New York, 1979. MR535766 (81d:12011)

[12] D.R. Hayes, A brief introduction to Drinfeld modules, The Arithmetic of Function Fields (D. Goss, D.R. Hayes, and M.I. Rosen, eds.), pp. 1-32, W. de Gruyter, Berlin, 1992. MR:1196509 (93m:11050)

[13] K. Lauter, A formula for constructing curves over finite fields with many rational points, $J$. Number Theory 74, 56-72 (1999). MR1670536 (99k:11088)

[14] S. Ling and C.P. Xing, Coding Theory. A First Course, Cambridge, 2004. MR2048591 (2005c:94001)

[15] H. Niederreiter and C.P. Xing, Rational points on curves over finite fields: Theory and Applications, London Mathematical Society Lecture Note Series 285, Cambridge, 2001. MR.1837382 (2002h:11055)

[16] H. Niederreiter, C.P. Xing, and K.Y. Lam, A new construction of algebraic-geometry codes, Applicable Algebra Engrg. Comm. Comput. 9, 373-381 (1999). MR1697176 (2000j:94044)

[17] F. Özbudak and H. Stichtenoth, Constructing codes from algebraic curves, IEEE Trans. Inform. Theory 45, 2502-2505 (1999). MR 1725138

[18] J.-P. Serre, "Local Fields," Springer, New York, 1979. MR.554237 (82e:12016)

[19] J.-P. Serre, "Rational points on curves over finite fields," Lecture Notes, Harvard University, 1985.

[20] H. Stichtenoth, Algebraic Function Fields and Codes, Springer, Berlin, 1993. MR1251961 (94k:14016)

[21] M.A. Tsfasman and S.G. Vladut, Algebraic-Geometric Codes, Kluwer, Dordrecht, 1991. MR.1186841 (93i:94023)

[22] G. van der Geer and M. van der Vlugt, Tables of curves with many points, 17 November, 2007, Website: http://www.science.uva.nl/ geer.

[23] C.P. Xing, Linear codes from narrow ray class groups of algebraic curves, IEEE Trans. on Information Theory, 50, 541-543(2004). MR2045029 (2005a:94108)

[24] C.P. Xing, H. Niederreiter, and K.Y. Lam, Constructions of algebraic-geometry codes, IEEE Trans. Inform. Theory 45, 1186-1193 (1999). MR1686251(2000g:94056)

[25] C.P. Xing, H. Niederreiter, and K.Y. Lam, A generalization of algebraic-geometry codes, IEEE Trans. Inform. Theory 45, 2498-2501 (1999). MR1725137

[26] C.P. Xing and S. Ling, A class of linear codes with good parameters, IEEE Trans. Inform. Theory 46, 2184-2188 (2000). MR1781376 (2001e:94018)

School of Physical and Mathematical Sciences, Nanyang Technological University, Singapore 637616 , Republic of Singapore.

E-mail address: xingcp@ntu.edu.sg

Systems \& Security Department (SSD), Institute for Infocomm Research (I2R), SinGApore 119613, Republic of Singapore.

E-mail address: slyeo@i2r.a-star.edu.sg 